\title{
N-stability of the $\theta$-method for reaction-diffusion problems
}

\author{
I. Fekete and I. Faragó
}




\title{
N-STABILITY OF THE $\theta$-METHOD FOR REACTION-DIFFUSION PROBLEMS
}

\author{
I. FEKETE AND I. FARAGÓ
}

Received 24 September, 2013

\begin{abstract}
In this paper we investigate the N-stability notion in an abstract Banach space setting. The main result is that thanks to this notion we have an alternative opportunity for verifying the stability of the numerical solution for periodic initial-value reaction-diffusion problems.
\end{abstract}

2010 Mathematics Subject Classification: 65J05; 65J15; 65M12

Keywords: numerical method, nonlinear problems, stability

\section{INTRODUCTION}

Many phenomena in nature such as physical, biological or chemical processes can be described by mathematical models. In chemistry one of the most investigated problems is the reaction-diffusion problem. Reaction-diffusion is a process in which two or more chemicals diffuse over a surface and react with one another to produce stable patterns. However (in general), the solution of such models cannot be given in a closed form, therefore we construct numerical models in order to approximate the exact solution. Generally, consistency in itself is not enough for convergence. To guarantee this property we introduce the notion of stability.

The stability property is verified for periodic initial-value reaction-diffusion problems in case of globally Lispchitz continuous forcing function $f$ in several works, e.g. in Ascher [1] and Thomas [8]. Regarding the stability proof, these books use the fact that we know the eigenvalues of the standard matrix replacement of the second derivative operator with periodic boundary conditions. Further techniques are introduced e.g., discrete time Fourier transform [8].

In the recent years we dealt with the investigation of the numerical solution of nonlinear operator equations in an abstract (Banach space) setting. This work has been summarized in $[3,4]$. In this paper our primary aim is to describe the discretizated version of the above mentioned problem and verify the $\mathrm{N}$-stability in an abstract setting. Thanks to this method we can offer a different approach for verifying the stability of nonlinear equations.

The work of the second author was supported by the Hungarian Research Grant OTKA K67819. 
This paper is organized as follows. In Section 2 we give a short mathematical formulation of the general description of mathematical and numerical models. Moreover, we define the basic numerical notions (convergence, consistency). In Section 3 we introduce the notion of $\mathrm{N}$-stability based on the work of Sans-Serna $([6,7])$. In Section 3.1 we describe the connection with the notion of linear stability. In Section 3.2 we verify that the $\theta$-method is N-stable both with and without the forcing term. In Section 4 we make some remarks and we draw conclusions.

\section{MATHematicAl BACKGROUND}

We consider the problem

$$
F(u)=0,
$$

where $F: \mathscr{D} \rightarrow \mathcal{Y}$ is a (nonlinear) operator, $\mathscr{D} \subset \mathcal{X}, \mathcal{X}$ and $\mathcal{Y}$ are normed spaces. In the theory of numerical analysis it is usually assumed that there exists a unique solution, which will be denoted by $\bar{u}$.

Definition 1. Problem (2.1) can be identified as a triplet $\mathscr{P}=(\mathcal{X}, \mathcal{Y}, F)$. We will refer to it as problem $\mathscr{P}$.

Definition 2. The sequence $\mathscr{N}=\left(\mathcal{X}_{n}, y_{n}, F_{n}\right)_{n \in \mathbb{N}}$ is called numerical method if it generates a sequence of problems

$$
F_{n}\left(u_{n}\right)=0, \quad n=1,2, \ldots,
$$

where

- $\mathcal{X}_{n}, y_{n}$ are normed spaces,

- $\mathscr{D}_{n} \subset \mathcal{X}_{n}$ and $F_{n}: \mathscr{D}_{n} \rightarrow Y_{n}$.

If there exists a unique solution of (2.2), it will be denoted by $\bar{u}_{n}$.

Remark 1. In the sequel we always assume the existence of $\bar{u}$ and $\bar{u}_{n}$.

Definition 3. We say that the sequence $\mathscr{D}=\left(\varphi_{n}, \psi_{n}, \Phi_{n}\right)_{n \in \mathbb{N}}$ is a discretization of problem $\mathscr{P}$ by numerical method $\mathscr{N}$, if

- the $\varphi_{n}$-s (respectively $\psi_{n}$-s) are restriction operators from $\mathcal{X}$ into $\mathcal{X}_{n}$ (respectively from $y_{\text {into }} y_{n}$ ),

- $\Phi_{n}:\{F: \mathscr{D} \rightarrow \mathcal{Y} \mid \mathscr{D} \subset \mathcal{X}\} \rightarrow\left\{F_{n}: \mathscr{D}_{n} \rightarrow \mathcal{Y}_{n} \mid \mathscr{D}_{n} \subset \mathcal{X}_{n}\right\}$.

In the sense of Definitions 1, 2 and 3 we can illustrate the general scheme, shown in Figure 1.

In our paper we always make the following assumption for the general discretization $\mathscr{D}$.

\section{Assumption 1.}

(a) The discretization $\mathscr{D}$ possesses the property $\psi_{n}(0)=0$.

(b) The discretization $\mathscr{D}$ generates the numerical method $\mathscr{N}$ with the property $\operatorname{dim} \mathcal{X}_{n}=\operatorname{dim} y_{n}<\infty$. 


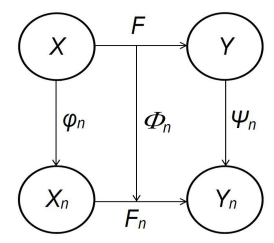

FIGURE 1. The general scheme of numerical methods.

(c) $F_{n}$ is continuous on some ball $B_{R}\left(\varphi_{n}(\bar{u})\right)$.

Remark 2. Obviously, when $\psi_{n}$ are linear operators, then Assumption 1 (a) is automatically satisfied.

Definition 4. The element $e_{n}=\varphi_{n}(\bar{u})-\bar{u}_{n} \in \mathcal{X}_{n}$ is called global discretization error. if

Definition 5. The discretization $\mathscr{D}$ applied to the problem $\mathscr{P}$ is called convergent holds.

$$
\lim \left\|e_{n}\right\| x_{n}=0
$$

Definition 6. The element $l_{n}(v)=F_{n}\left(\varphi_{n}(v)\right)-\psi_{n}(F(v)) \in y_{n}$ is called local discretization error at the element $v \in \mathcal{D}$. The local discretization error on the solution, i.e., $l_{n}(\bar{u})=F_{n}\left(\varphi_{n}(\bar{u})\right)-\psi_{n}(F(\bar{u}))=F_{n}\left(\varphi_{n}(\bar{u})\right)$ is called local discretization error.

Definition 7. The discretization $\mathscr{D}$ applied to problem $\mathscr{P}$ is called consistent on the element $v \in D$ if

- $\varphi_{n}(v) \in \mathscr{D}_{n}$ holds from some index,

- for these elements the relation

$$
\lim \left\|l_{n}(v)\right\|_{y_{n}}=0
$$

holds.

\section{N-STABILITY AND ITS ADVANTAGES}

In numerical analysis one of the most important task is to guarantee the convergence of the sequence of the numerical solutions. Generally, consistency in itself is not enough for convergence. To guarantee this property we introduce the notion of stability.

According to Definition 4, the convergence yields that the global discretization error $e_{n}$ tends to zero. Having consistency, we have information about the local discretization error, only. Intuitively, this means the following requirement. When $l_{n}(\bar{u})=F_{n}\left(\varphi_{n}(\bar{u})\right)-F\left(\bar{u}_{n}\right)$ is small, then $e_{n}=\varphi(\bar{u})-\bar{u}_{n}$ should be small, too. 
Because $\bar{u}$ is unknown, therefore in first approach we require this property for any pairs in $\mathscr{D}_{n}$, i.e., for all $z_{n}, w_{n} \in \mathscr{D}_{n}$ we require the inequality

$$
\left\|z_{n}-w_{n}\right\|_{x_{n}} \leq S\left\|F_{n}\left(z_{n}\right)-F_{n}\left(w_{n}\right)\right\|_{y_{n}},
$$

where the stability constant $S$ is indepedent of the mesh-size parameter.

This idea leads to make the first attempt to define the nonlinear stability notion.

Definition 8. The discretization $\mathscr{D}$ is called $N$-stable on the problem $\mathscr{P}$ if there exists a positive stability constant $S$, such that for each $z_{n}, w_{n} \in \mathscr{D}_{n}$, the estimation (3.1) holds.

Furthermore we will refer to this notion as the naive stability (N-stability).

Theorem 1. We assume that

- there exists the solution of the problem (2.1)-(2.2),

- the discretization $\mathscr{D}$ is consistent and $N$-stable at $\bar{u}$ with constant $S$ on problem $\mathscr{P}$.

Then the discretization $\mathscr{D}$ is convergent on problem $\mathscr{P}$.

Proof. Due to the N-stability, we have the relation

$$
\left\|e_{n}\right\|_{x_{n}}=\left\|\varphi_{n}(\bar{u})-\bar{u}_{n}\right\|_{X_{n}} \leq S\|F_{n}\left(\varphi_{n}(\bar{u})\right)-\underbrace{F_{n}\left(\bar{u}_{n}\right)}_{=0}\| y_{n}
$$

which leads to the estimation

$$
\left\|e_{n}\right\|_{x_{n}} \leq S\left\|F_{n}\left(\varphi_{n}(\bar{u})\right)\right\|_{y_{n}}=S\left\|l_{n}(\bar{u})\right\|_{y_{n}}
$$

and hence, for consistent methods this implies the convergence.

Remark 3. Formally, this statement can be written as the "basic theory of numerical analysis":

$$
\text { Consistency }+ \text { Stability } \Rightarrow \text { Convergence }
$$

\subsection{Linear stability}

For studying the nonlinear stability, it is expedient to present the linear case. In this case the consistency and the stability together ensure the convergence. This result is well known as the Lax (or sometimes Lax-Richtmyer-Kantorovich [5]) theorem. From the formulation of the main theorem it turns out that these two, directly checkable conditions (i.e., the consistency and stability) serve together the convergence. Let

$$
L_{n}\left(u_{n}\right)=0, \quad n=1,2, \ldots,
$$

where $L_{n}$ is a linear oparator and $L_{n}: \mathscr{D}_{n} \rightarrow y_{n}$. 
Definition 9. The discretization $\mathscr{D}$ is called stable on the problem $\mathscr{P}$ if there exists positive stability constant $S$, such that for each $v_{n} \in \mathscr{D}_{n}$

$$
\left\|v_{n}\right\|_{X_{n}} \leq S\left\|L_{n}\left(v_{n}\right)\right\|_{y_{n}}
$$

holds.

The bound (3.3) implies three basic properties:

i, For any problems (3.2), the relation (3.3) shows that $L_{n}\left(v_{n}\right)=0$ implies that $v_{n}=0$, i.e., $L_{n}$ is injective and hence $L_{n}^{-1}$ exists on the entire space $y_{n}$ by Assumption 1 (b). Therefore, the stability bound implies the existence and uniqueness of solutions of (3.2).

ii, Due to the previous property i, using the estimate (3.3) we have

$$
\left\|L_{n}^{-1} s_{n}\right\|_{x_{n}} \leq S\left\|s_{n}\right\|_{y_{n}}
$$

for arbitrary $s_{n} \in \mathcal{Y}_{n}$. Therefore the uniform norm estimation

$$
\left\|L_{n}^{-1}\right\|_{\operatorname{Lin}\left(y_{n}, x_{n}\right)} \leq S
$$

holds.

iii, In view of (3.3), we obtain the "basic theory of numerical analysis". In fact, due to the linearity of $L_{n}$, by the choice $e_{n}=\varphi_{n}(\bar{u})-\bar{u}_{n}$, we have

$$
\left\|\varphi_{n}(\bar{u})-\bar{u}_{n}\right\|_{\mathcal{X}_{n}} \leq S\left\|L_{n}\left(\varphi_{n}(\bar{u})\right)-L_{n}\left(\bar{u}_{n}\right)\right\|_{y_{n}}
$$

which leads to the estimation

$$
\left\|e_{n}\right\|_{X_{n}}=\left\|\varphi_{n}(\bar{u})-\bar{u}_{n}\right\|_{X_{n}} \leq S\left\|l_{n}(\bar{u})\right\|_{y_{n}}
$$

and for consistent methods, this implies the convergence.

\section{Remark 4.}

(a) Definition 9 is special case of Definition 8 with the choice $F_{n}\left(u_{n}\right)=L_{n}\left(u_{n}\right)$,

(b) The reverse of property ii, is true. Namely, if the inverse of the linear operators $L_{n}$ exist and they are uniformly bounded, then they are N-stable, too.

(c) In a straightforward way the above mentioned three basic properties can be generalized for the nonhomogeneous linear equation $F_{n}\left(u_{n}\right) \equiv L_{n}\left(u_{n}\right)-$ $b_{n}=0$.

\section{2. $N$-stability of the reaction-diffusion problem}

We showed in the previous section that $\mathrm{N}$-stability is equivalent to the regular stability notion in the linear case. In addition, this notion has an advantageous property for nonlinear equations as well. Namely, it is useful from the application point of view. In the rest of the paper our aim is to verify the $\mathrm{N}$-stability of the reactiondiffusion problem under certain conditions using the $\theta$-method. 


\subsubsection{Reaction-diffusion problem without forcing term}

Consider the following periodic initial-value reaction-diffusion problem:

$$
\begin{gathered}
\partial_{t} u(t, x)=\partial_{x x} u(t, x), \quad x \in \mathbb{R}, t \in[0, T], \\
u(t, x)=u(t, x+1), \quad x \in \mathbb{R}, t \in[0, T], \\
u(0, x)=u^{0}(x), \quad x \in \mathbb{R},
\end{gathered}
$$

where $T \in \mathbb{R}^{+}$. The conditions (3.5)-(3.6) are periodic boundary conditions and initial-value conditions, where $u^{0}$ is a given one-periodic function. It is easy to see that the continuous problem (3.4)-(3.6) can be rewritten in the form of (2.1). As we have mentioned, we assume the existence of unique, sufficiently smooth solution of the problem (3.4)-(3.6).

Remark 5. Since the solution is periodic, it is sufficient to determine the solution in one period, only.

To create the discretization $\mathscr{D}$ on the above mentioned problem we define both the spatial and time grids, as follows. The spatial grid points are

$$
\left\{x_{j}=j h, \text { where } j=1, \ldots, n, h=1 / n \text { and } n \in \mathbb{N}, n \geq 2\right\},
$$

and the time levels are

$$
\left\{t_{k}=k \delta, \text { where } k=0, \ldots, K \text { and } \delta=T / K\right\} .
$$

Applying the $\theta$-method to this reaction-diffusion problem, for $\theta \in[0,1], j=1, \ldots, n$, and $k=0, \ldots, K-1$, we gain

$$
\frac{u_{j}^{k+1}-u_{j}^{k}}{\delta}-(1-\theta) \frac{u_{j-1}^{k}-2 u_{j}^{k}+u_{j+1}^{k}}{h^{2}}-\theta \frac{u_{j-1}^{k+1}-2 u_{j}^{k+1}+u_{j+1}^{k+1}}{h^{2}}=0,
$$

where using the periodic boundary conditions it is obvious that $u_{0}^{k}=u_{n}^{k}, u_{1}^{k}=$ $u_{n+1}^{k}, u_{0}^{k+1}=u_{n}^{k+1}$ and $u_{1}^{k+1}=u_{n+1}^{k+1}$. The initial-value condition can be written as

$$
u_{j}^{0}-u^{0}\left(x_{j}\right)=0, \quad j=1, \ldots, n .
$$

In the next step is that we rewrite (3.7)-(3.8) in the form of (2.2). To this aim we define the vector space of the grid functions $\mathcal{K}_{n}$, defined on the grid points $x_{j}: 1 \leq j \leq n$. If we consider $u_{j}^{k}$ for the time level $t_{k}$ for each $k$, then the denoted vector is $\mathbf{u}^{k} \in \mathcal{K}_{n}$. In Definition 3 we define $\varphi_{n}, \psi_{n}$ as the grid restriction operators. Hence, (3.7)-(3.8) can be written as

$$
\begin{gathered}
\frac{\mathbf{u}^{k+1}-\mathbf{u}^{k}}{\delta}-(1-\theta) D_{p}^{2} \mathbf{u}^{k}-\theta D_{p}^{2} \mathbf{u}^{k+1}=0, \quad k=0, \ldots, K-1, \\
\mathbf{u}^{0}-\varphi_{n}\left(u^{0}\right)=0,
\end{gathered}
$$


where $\mathbf{u}^{0}=\left(u^{0}\left(x_{1}\right), \ldots, u^{0}\left(x_{n}\right)\right) \in \mathcal{K}_{n}$ and $D_{p}^{2}$ denotes the standard discretization matrix with periodic boundary conditions, i.e.,

$$
D_{p}^{2}=\frac{1}{h^{2}}\left(\begin{array}{rrrrrrr}
-2 & 1 & 0 & \cdots & 0 & 0 & 1 \\
1 & -2 & 1 & 0 & \cdots & 0 & 0 \\
0 & 1 & -2 & 1 & 0 & \cdots & 0 \\
\vdots & \ddots & \ddots & \ddots & \ddots & \ddots & \vdots \\
0 & \cdots & 0 & 1 & -2 & 1 & 0 \\
0 & \cdots & \cdots & 0 & 1 & -2 & 1 \\
1 & 0 & 0 & \cdots & 0 & 1 & -2
\end{array}\right)
$$

We choose in Definition 2: $X_{n}=y_{n}=\underbrace{\mathcal{K}_{n} \times \ldots \times \mathcal{K}_{n}}_{K+1}$, hence $\mathbf{v}_{n}:=\left(\mathbf{v}^{0}, \ldots, \mathbf{v}^{K}\right) \in$ $X_{n}$. We introduce the following norms:

- in $\mathcal{K}_{n}:\left\|\mathbf{v}^{k}\right\|_{\mathcal{K}_{n}}=\max _{1 \leq j \leq n}\left|v^{k}\left(x_{j}\right)\right|=\left\|\mathbf{v}^{k}\right\|_{\infty}$,

- in $\mathcal{X}_{n}:\left\|\mathbf{v}_{n}\right\|_{\mathcal{X}_{n}}=\max _{0 \leq k \leq K}\left\|\mathbf{v}^{k}\right\|_{\mathcal{K}_{n}}$,

- in $y_{n}:\left\|\mathbf{v}_{n}\right\|_{y_{n}}=\left\|\mathbf{v}^{0}\right\|_{\mathcal{K}_{n}}+\sum_{j=1}^{K} \delta\left\|\mathbf{v}^{j}\right\|_{\mathcal{K}_{n}}$.

Let $\mathbf{v}_{n} \in \mathcal{X}_{n}$ any element and we denote by $\eta_{n}=\left(\eta^{0}, \ldots, \eta^{K}\right) \in \mathcal{Y}_{n}$ its image. Then the mapping $F_{n}: \mathcal{X}_{n} \rightarrow \mathcal{Y}_{n}$ can be written as $F_{n}\left(\mathbf{v}_{n}\right)=\eta_{n}$. Particularly, for our discretization (3.9)-(3.10) it yields the following:

$$
\begin{gathered}
\frac{\mathbf{v}^{k+1}-\mathbf{v}^{k}}{\delta}-(1-\theta) D_{p}^{2} \mathbf{v}^{k}-\theta D_{p}^{2} \mathbf{v}^{k+1}=\eta^{k+1}, k=0, \ldots, K-1, \\
\mathbf{v}^{0}=\eta^{0} .
\end{gathered}
$$

The investigated method can be rewritten in the form

$$
\mathbf{v}^{k+1}=Q_{1}^{-1} Q_{2} \mathbf{v}^{k}+\delta Q_{1}^{-1} \eta^{k+1}
$$

where $Q_{1}=I-\theta \delta D_{p}^{2}$ and $Q_{2}=I+(1-\theta) \delta D_{p}^{2}$ are the subtransition matrices (which depend on $h$ and $\delta$ ). Introducing the notation $r=\delta / h^{2}$ we can write $Q_{1}$ as

$$
Q_{1}=\left(\begin{array}{ccccccc}
1+2 r \theta & -r \theta & 0 & \ldots & 0 & 0 & -r \theta \\
-r \theta & 1+2 r \theta & -r \theta & 0 & \ldots & 0 & 0 \\
0 & -r \theta & 1+2 r \theta & -r \theta & 0 & \ldots & 0 \\
\vdots & \ddots & \ddots & \ddots & \ddots & \ddots & \vdots \\
0 & \ldots & 0 & -r \theta & 1+2 r \theta & -r \theta & 0 \\
0 & \ldots & \ldots & 0 & -r \theta & 1+2 r \theta & -r \theta \\
-r \theta & 0 & 0 & \ldots & 0 & -r \theta & 1+2 r \theta
\end{array}\right) .
$$


Definition 10. An $A \in \mathbb{R}^{n \times n}$ matrix is an M-matrix, if

- $a_{i j} \leq 0$ for all $i \neq j$,

- there exists a vector $g \in \mathbb{R}^{n}, g>0$ such that $A g>0$.

Theorem 2. Let $A \in \mathbb{R}^{n \times n}$ be an M-matrix. Then

$$
\left\|A^{-1}\right\|_{\infty} \leq \frac{\|g\|_{\infty}}{\min _{1 \leq i \leq n}(A g)_{i}}
$$

Proof. It can be found in [2].

Since $r>0$ and $\theta \in[0,1]$, thus $Q_{1}$ is strictly diagonally dominant and $\left(Q_{1}\right)_{i j} \leq 0$ for all $i \neq j$. Hence, $Q_{1}$ is an M-matrix with $g=(1, \ldots, 1)^{T}$. Due to (3.12), we have the estimate

$$
\left\|Q_{1}^{-1}\right\|_{\infty} \leq \frac{\|g\|_{\infty}}{\min _{1 \leq i \leq n}\left(Q_{1}^{-1} g\right)_{i}}=\frac{1}{1+2 r \delta-r \delta-r \delta}=1 .
$$

The matrix $Q_{2}$ can be written in the form

$$
Q_{2}=\left(\begin{array}{ccccccc}
a & b & 0 & \cdots & 0 & 0 & b \\
b & a & b & 0 & \cdots & 0 & 0 \\
0 & b & a & b & 0 & \cdots & 0 \\
\vdots & \ddots & \ddots & \ddots & \ddots & \ddots & \vdots \\
0 & \cdots & 0 & b & a & b & 0 \\
0 & \cdots & \cdots & 0 & b & a & b \\
b & 0 & 0 & \cdots & 0 & b & a
\end{array}\right),
$$

where $a=1-2 r(1-\theta)$ and $b=r(1-\theta)$. It is easy to see that under the assumption $r \leq \frac{1}{2(1-\theta)}$ we have

$$
\left\|Q_{2}\right\|_{\infty}=1
$$

Introducing the notation $Q=Q_{1}^{-1} Q_{2}$, the iteration (3.11) can be written as

$$
\mathbf{v}^{k+1}=Q \mathbf{v}^{k}+\delta Q_{1}^{-1} \eta^{k+1} .
$$

Applying the above recursion and putting $\mathbf{v}^{0}=\eta^{0}$ for any $k=0,1, \ldots, K$, we get

$$
\mathbf{v}^{k}=Q^{k} \mathbf{v}^{0}+\sum_{j=1}^{k} \delta Q^{j-1} Q_{1}^{-1} \eta^{k+1-j}=Q^{k} \eta^{0}+\delta Q^{k-1} Q_{1}^{-1} \eta^{1}+\ldots+\delta Q_{1}^{-1} \eta^{k} .
$$

Hence, according to the introduced norms, we obtain the estimation

$$
\left\|\mathbf{v}_{n}\right\|_{X_{n}} \leq \max _{0 \leq k \leq K}\left\{\max _{1 \leq j \leq k}\left\{\left\|Q^{k}\right\|_{\infty},\left\|Q^{j-1} Q_{1}^{-1}\right\|_{\infty}\right\}\right\}\left\|\eta_{n}\right\|_{y_{n}} .
$$

Using the relations (3.13) and (3.14), we get that

$$
\|Q\|_{\infty}=\left\|Q_{1}^{-1} Q_{2}\right\|_{\infty} \leq\left\|Q_{1}^{-1}\right\|_{\infty}\left\|Q_{2}\right\|_{\infty} \leq 1,
$$


thus $\left\|Q^{k}\right\|_{\infty} \leq\|Q\|_{\infty}^{k} \leq 1$ and similarly $\left\|Q^{j-1} Q_{1}^{-1}\right\|_{\infty} \leq\|Q\|_{\infty}^{j-1}\left\|Q_{1}^{-1}\right\|_{\infty} \leq 1$. Hence, obviously we have

$$
\max _{0 \leq k \leq K}\left\{\max _{1 \leq j \leq k}\left\{\left\|Q^{k}\right\|_{\infty},\left\|Q^{j-1} Q_{1}^{-1}\right\|_{\infty}\right\}\right\}=1 .
$$

Since $F_{n}\left(\mathbf{v}_{n}\right)=\eta_{n}$, we can rewrite (3.15) as

$$
\left\|\mathbf{v}_{n}\right\|_{X_{n}} \leq\left\|\eta_{n}\right\|_{y_{n}}=\left\|F_{n}\left(\mathbf{v}_{n}\right)\right\|_{y_{n}} .
$$

For any elements $\mathbf{z}_{n}, \mathbf{w}_{n} \in \mathcal{X}_{n}$ we denote by $\varrho_{n}$ and $\xi_{n}$ their image, i.e., $F_{n}\left(\mathbf{z}_{n}\right)=\varrho_{n}$ and $F_{n}\left(\mathbf{w}_{n}\right)=\xi_{n}$. This results in the relations

$$
\begin{aligned}
\frac{\mathbf{z}^{k+1}-\mathbf{z}^{k}}{\delta}-(1-\theta) D_{p}^{2} \mathbf{z}^{k}-\theta D_{p}^{2} \mathbf{z}^{k+1} & =\varrho^{k+1}, k=0, \ldots, K-1, \\
\mathbf{z}^{0}-\varphi_{n}\left(u^{0}\right) & =\varrho^{0}, \\
\frac{\mathbf{w}^{k+1}-\mathbf{w}^{k}}{\delta}-(1-\theta) D_{p}^{2} \mathbf{w}^{k}-\theta D_{p}^{2} \mathbf{w}^{k+1} & =\xi^{k+1}, k=0, \ldots, K-1, \\
\mathbf{w}^{0}-\varphi_{n}\left(u^{0}\right) & =\xi^{0} .
\end{aligned}
$$

Substracting (3.18) from (3.17), we gain

$$
\mathbf{z}^{k+1}-\mathbf{w}^{k+1}=Q\left(\mathbf{z}^{k}-\mathbf{w}^{k}\right)+\delta Q_{1}^{-1}\left(\varrho^{k+1}-\xi^{k+1}\right), \quad k=0, \ldots, K-1 .
$$

Using (3.16) by the notation $\mathbf{v}_{n}=\mathbf{z}_{n}-\mathbf{w}_{n}$, we gain

$$
\left\|\mathbf{z}_{n}-\mathbf{w}_{n}\right\|_{X_{n}} \leq\left\|\varrho_{n}-\xi_{n}\right\|_{y_{n}}=\left\|F_{n}\left(\mathbf{z}_{n}\right)-F_{n}\left(\mathbf{w}_{n}\right)\right\| y_{n} .
$$

It is easy to see that the above estimation is in the form of (3.1) with $S=1$. Hence, under the condition $r \leq \frac{1}{2(1-\theta)}$ the reaction-diffusion problem is N-stable. Hence, the following statement is true.

Theorem 3. Under the condition $r \leq \frac{1}{2(1-\theta)}$ the $\theta$-method is convergent for the periodic initial-value reaction-diffusion problem (3.4)-(3.6).

Proof. The $\theta$-method is consistent and $\mathrm{N}$-stable for this problem. Hence, due to Theorem 1, it is convergent, too.

\subsubsection{Reaction-diffusion problem with forcing term}

Further we consider the following periodic initial-value reaction-diffusion problem:

$$
\begin{gathered}
\partial_{t} u(t, x)=\partial_{x x} u(t, x)+f(u), \quad x \in \mathbb{R}, t \in[0, T], \\
u(t, x)=u(t, x+1), \quad x \in \mathbb{R}, t \in[0, T], \\
u(0, x)=u^{0}(x), \quad x \in \mathbb{R},
\end{gathered}
$$

where $T \in \mathbb{R}^{+}$. In equation (3.19) we assume that $f: \mathbb{R} \rightarrow \mathbb{R}$ is a given globally Lipschitz continuous function. The conditions (3.20)-(3.21) are periodic boundary conditions and initial-value conditions, where $u^{0}$ is a given one-periodic function. It 
is easy to see that the continuous problem (3.19)-(3.21) can be rewritten in the form of (2.1). As we have mentioned, we assume the existence of unique, sufficiently smooth solution of the problem (3.19)-(3.21).

Remark 6. Since the solution is periodic, it is sufficient to determine the solution in one period, only.

Let us take the formerly introduced spatial and time grids and normes. We apply the IMEX-method based on the previous train of thought. For any elements $\mathbf{z}_{n}, \mathbf{w}_{n} \in \mathcal{X}_{n}$ we denote by $\varrho_{n}$ and $\xi_{n}$ their image, i.e., $F_{n}\left(\mathbf{z}_{n}\right)=\varrho_{n}$ and $F_{n}\left(\mathbf{w}_{n}\right)=\xi_{n}$. Then we consider the following two problems:

$$
\begin{gathered}
\frac{\mathbf{z}^{k+1}-\mathbf{z}^{k}}{\delta}-(1-\theta) D_{p}^{2} \mathbf{z}^{k}-\theta D_{p}^{2} \mathbf{z}^{k+1}-\mathbf{f}\left(\mathbf{z}^{k}\right)=\varrho^{k+1}, k=0, \ldots, K-1, \\
\mathbf{z}^{0}-\varphi_{n}\left(u^{0}\right)=\varrho^{0}, \\
\frac{\mathbf{w}^{k+1}-\mathbf{w}^{k}}{\delta}-(1-\theta) D_{p}^{2} \mathbf{w}^{k}-\theta D_{p}^{2} \mathbf{w}^{k+1}-\mathbf{f}\left(\mathbf{w}^{k}\right)=\xi^{k+1}, k=0, \ldots, K-1, \\
\mathbf{w}^{0}-\varphi_{n}\left(u^{0}\right)=\xi^{0},
\end{gathered}
$$

where $\mathbf{f}$ denotes the grid function defined on the grid points $x_{j}$, i.e., $\left[\mathbf{f}\left(\mathbf{z}^{k}\right)\right]_{j}=$ $\varphi_{n}\left(f\left(x_{j}\right)\right)$ for all $j=1, \ldots, n$. Substracting (3.23) from (3.22), for $k=0, \ldots, K-1$, we get

$$
\mathbf{z}^{k+1}-\mathbf{w}^{k+1}=Q_{1}^{-1} Q_{2}\left(\mathbf{z}^{k}-\mathbf{w}^{k}\right)+\delta Q_{1}^{-1}\left(\mathbf{f}\left(\mathbf{z}^{k}\right)-\mathbf{f}\left(\mathbf{w}^{k}\right)\right)+\delta Q_{1}^{-1}\left(\varrho^{k+1}-\xi^{k+1}\right),
$$

where $Q_{1}$ and $Q_{2}$ are the earlier defined subtransition matrices. Since $f$ is a given globally Lipschitz continuous function, this implies

$$
\left\|\mathbf{f}\left(\mathbf{z}^{k}\right)-\mathbf{f}\left(\mathbf{w}^{k}\right)\right\|_{\mathcal{K}_{n}} \leq L\left\|\mathbf{z}^{k}-\mathbf{w}^{k}\right\|_{\mathcal{K}_{n}} .
$$

Using the Lipschitz property (3.25) and applying the results (3.13) and (3.14) respectively, we get $\left\|Q_{1}^{-1} Q_{2}\right\|_{\infty} \leq 1$. Then recursion (3.24) shows that for $k=0, \ldots, K$ we have the estimate

$$
\left\|\mathbf{z}^{k}-\mathbf{w}^{k}\right\|_{\mathcal{K}_{n}} \leq(1+\delta L)\left\|\mathbf{z}^{k-1}-\mathbf{w}^{k-1}\right\|_{\mathcal{K}_{n}}+\delta\left\|\varrho^{k}-\xi^{k}\right\|_{\mathcal{K}_{n}} .
$$

Applying recursion (3.26) and $\mathbf{z}^{0}-\mathbf{w}^{0}=\varrho^{0}-\xi^{0}$, we get for $k=0, \ldots, K$

$$
\left\|\mathbf{z}^{k}-\mathbf{w}^{k}\right\|_{\mathcal{K}_{n}} \leq(1+\delta L)^{k}\left\|\varrho_{n}-\xi_{n}\right\| y_{n} .
$$

Using that $\delta K=T$, we get the estimation

$$
\left\|\mathbf{z}_{n}-\mathbf{w}_{n}\right\|_{X_{n}} \leq(1+\delta L)^{K}\left\|\varrho_{n}-\xi_{n}\right\|_{y_{n}} \leq e^{L T}\left\|F_{n}\left(\mathbf{z}_{n}\right)-F_{n}\left(\mathbf{w}_{n}\right)\right\|_{y_{n}} .
$$

It is easy to see that the above estimation is in the form of (3.1) with $S=e^{L T}$. Hence, under the assumptions made, the reaction-diffusion problem is $\mathrm{N}$-stable. 
Theorem 4. Under the condition $r \leq \frac{1}{2(1-\theta)}$, for the Lipschitzian forcing term $f$ the $\theta$-method is convergent for the periodic initial-value reaction-diffusion problem (3.19)-(3.21).

Proof. The $\theta$-method is consistent and $\mathrm{N}$-stable for this problem. Hence, due to Theorem 1, it is convergent, too.

Remark 7. Due to these results we automatically get

(a) both the explicit finite difference method (for $\theta=0$ ) under the condition $r \leq$ 0.5 and the IMEX-method (for $\theta=1$ ) without any condition are $\mathrm{N}$-stable,

(b) if the given forcing term is in the form of $f(t, u)$ and it is a Lipschitz continuous function with respect to its second variable by the constant $L$, then we can similarly verify that the $\theta$-method is convergent for the investigated problem.

\section{SUMMARY}

In this paper we investigated the reaction-diffusion problem under certain conditions in an abstract setting. The base of our framework was the N-stablity notion. As we have shown in Section 3, it is a useful notion regarding both theoretical results and applications. We verified that the $\theta$-method is $\mathrm{N}$-stable for the reaction-diffusion problem both with and without the forcing term.

The main advantage of this notion is that we can offer another tool for verifying the stability property for nonlinear equations. In the near future it is worth dealing with the N-stability of different physical, biological or chemical processes.

\section{REFERENCES}

[1] U. M. Ascher, Numerical methods for evolutionary differential equations, ser. Computational Science \& Engineering. Society for Industrial and Applied Mathematics (SIAM), Philadelphia, PA, 2008, vol. 5. [Online]. Available: http://dx.doi.org/10.1137/1.9780898718911

[2] A. Berman and R. J. Plemmons, Nonnegative matrices in the mathematical sciences. Academic Press [Harcourt Brace Jovanovich, Publishers], New York-London, 1979, computer Science and Applied Mathematics.

[3] I. Faragó and I. Fekete, "T-stability of general one-step methods for abstract initial-value problems," Open Math. J., vol. 6, pp. 19-25, 2013.

[4] I. Faragó, M. E. Mincsovics, and I. Fekete, "Notes on the basic notions in nonlinear numerical analysis," in The 9th Colloquium on the Qualitative Theory of Differential Equations, ser. Proc. Colloq. Qual. Theory Differ. Equ. Electron. J. Qual. Theory Differ. Equ., Szeged, 2012, vol. 6, pp. 1-22.

[5] P. D. Lax and R. D. Richtmyer, "Survey of the stability of linear finite difference equations," Comm. Pure Appl. Math., vol. 9, pp. 267-293, 1956.

[6] J. C. López Marcos and J. M. Sanz-Serna, "A definition of stability for nonlinear problems," in Numerical treatment of differential equations (Halle, 1987), ser. Teubner-Texte Math. Teubner, Leipzig, 1988, vol. 104, pp. 216-226.

[7] J. M. Sanz-Serna, "Two topics in nonlinear stability," in Advances in numerical analysis, Vol. I (Lancaster, 1990), ser. Oxford Sci. Publ. Oxford Univ. Press, New York, 1991, pp. 147-174. 
[8] J. W. Thomas, Numerical partial differential equations: finite difference methods, ser. Texts in Applied Mathematics. Springer-Verlag, New York, 1995, vol. 22. [Online]. Available: http://dx.doi.org/10.1007/978-1-4899-7278-1

\section{Authors' addresses}

\section{Fekete}

Eötvös Loránd University, Department of Applied Analysis and Computational Mathematics, Pázmány P. s. 1/C, 1117 Budapest, Hungary

E-mail address: feipaatecs.elte.hu

\section{Faragó}

MTA-ELTE Numerical Analysis and Large Networks Research Group, Pázmány P. s. 1/C, 1117 Budapest, Hungary

E-mail address: faragoisecs.elte.hu 